PROCEEDINGS OF THE

AMERICAN MATHEMATICAL SOCIETY

Volume 140, Number 4, April 2012, Pages 1321-1330

S 0002-9939(2011)10980-8

Article electronically published on August 1,2011

\title{
UNIQUE CONTINUATION FOR DISCRETE NONLINEAR WAVE EQUATIONS
}

\author{
HELGE KRÜGER AND GERALD TESCHL
}

(Communicated by Walter Van Assche)

\begin{abstract}
We establish unique continuation for various discrete nonlinear wave equations. For example, we show that if two solutions of the Toda lattice coincide for one lattice point in some arbitrarily small time interval, then they coincide everywhere. Moreover, we establish analogous results for the Toda, Kac-van Moerbeke, and Ablowitz-Ladik hierarchies. Although all these equations are integrable, the proof does not use integrability and can be adapted to other equations as well.
\end{abstract}

\section{INTRODUCTION}

Unique continuation results for wave equations have a long tradition and seem to originate in control theory. One of the first results seems to be the one by Zhang [20, where he proves that if a short-range solution of the Korteweg-de Vries $(\mathrm{KdV})$ equation vanishes on an open subset in the $(x, t)$-plane, then it must vanish everywhere. Since then, this result has been extended in various directions and for different equations (see for example [1, the introduction in [10] for the case of the nonlinear Schrödinger equation, [5], 11, [12, for the generalized KdV equation, 14] for the Camassa-Holm equation).

However, all the results so far seem to only deal with wave equations which are continuous in the spatial direction and this clearly raises the question for such unique continuation results for wave equations which are discrete in the spatial variable. In particular, to the best of our knowledge, there are no results for example for the Toda equation, one of the most prominent discrete systems. While in principle the strategy from Zhang [20] would be applicable to the Toda lattice, it is the purpose of this paper to advocate a much simpler direct approach in the discrete case. We will start with the Toda lattice as our prototypical example and then show how the entire Toda hierarchy as well as the Kac-van Moerbeke and AblowitzLadik hierarchies can be treated. It is important to stress that our approach does not use integrability of these equations and hence can be adapted to more general systems. On the other hand, our approach is restricted to one dimension in the spatial variable and thus does not apply to the discrete Schrödinger equation on $\mathbb{Z}^{d}$.

Received by the editors April 1, 2009 and, in revised form, December 30, 2010.

2010 Mathematics Subject Classification. Primary 35L05, 37K60; Secondary 37K15, 37K10.

Key words and phrases. Unique continuation, Toda lattice, Kac-van Moerbeke lattice, Ablowitz-Ladik equations, discrete nonlinear Schrödinger equation, Schur flow.

Research supported by the Austrian Science Fund (FWF) under grant No. Y330 and the National Science Foundation (NSF) under grant No. DMS-0800100.

(C)2011 American Mathematical Society Reverts to public domain 28 years from publication 1321 
Due to the connections with localization for discrete Anderson-Bernoulli models, unique continuation for this model is an important open problem; see [2], 3].

\section{The Toda lattice}

In this section we want to treat the Toda lattice as the prototypical example. To this end, recall the Toda lattice [19] (in Flaschka's variables [7])

$$
\begin{aligned}
& \dot{a}(n, t)=a(n, t)(b(n+1, t)-b(n, t)), \\
& \dot{b}(n, t)=2\left(a(n, t)^{2}-a(n-1, t)^{2}\right), \quad n \in \mathbb{Z},
\end{aligned}
$$

where the dot denotes a derivative with respect to $t$. It is a well-studied physical model and one of the prototypical discrete integrable wave equations. We refer to the monographs [6], 16], [19] or the review articles [13, [17, for further information.

Theorem 2.1. Assume that $a_{0}(n, t), b_{0}(n, t)$ and $a(n, t), b(n, t)$ are complex-valued solutions of the Toda lattice (2.1) with $a_{0}(n, t) \neq 0$ for all $(n, t) \in \mathbb{Z} \times \mathbb{R}$ such that there is one $n_{0} \in \mathbb{Z}$ and two times $t_{0}<t_{1}$ such that

$$
a_{0}\left(n_{0}, t\right)^{2}=a\left(n_{0}, t\right)^{2}, \quad b_{0}\left(n_{0}, t\right)=b\left(n_{0}, t\right),
$$

for $t \in\left(t_{0}, t_{1}\right)$. Then

$$
a_{0}(n, t)^{2}=a(n, t)^{2}, \quad b_{0}(n, t)=b(n, t)
$$

for all $(n, t) \in \mathbb{Z} \times \mathbb{R}$.

Proof. It suffices to prove that (2.2) for $n_{0}$ implies (2.2) for $n_{0}-1$ and $n_{0}+1$. We start with $N_{0}-1$ and first observe that (2.1) implies that

$$
\begin{aligned}
0 & =\dot{b}\left(n_{0}, t\right)-\dot{b}_{0}\left(n_{0}, t\right)=2\left(a\left(n_{0}, t\right)^{2}-a_{0}\left(n_{0}, t\right)^{2}-a\left(n_{0}-1, t\right)^{2}+a_{0}\left(n_{0}-1, t\right)^{2}\right) \\
& =-2\left(a\left(n_{0}-1, t\right)^{2}-a_{0}\left(n_{0}-1, t\right)^{2}\right)
\end{aligned}
$$

and thus $a\left(n_{0}-1, t\right)^{2}=a_{0}\left(n_{0}-1, t\right)^{2}$. Using this we compute

$$
\begin{aligned}
0 & =\frac{\dot{a}\left(n_{0}-1, t\right)}{a\left(n_{0}-1, t\right)}-\frac{\dot{a}_{0}\left(n_{0}-1, t\right)}{a_{0}\left(n_{0}-1, t\right)} \\
& =b\left(n_{0}, t\right)-b_{0}\left(n_{0}, t\right)-b\left(n_{0}-1, t\right)+b_{0}\left(n_{0}-1, t\right) \\
& =-b\left(n_{0}-1, t\right)+b_{0}\left(n_{0}-1, t\right),
\end{aligned}
$$

so $b\left(n_{0}-1, t\right)=b_{0}\left(n_{0}-1, t\right)$. Now for $n_{0}+1$, we begin with

$$
\begin{aligned}
0 & =\frac{\dot{a}\left(n_{0}, t\right)}{a\left(n_{0}, t\right)}-\frac{\dot{a}_{0}\left(n_{0}, t\right)}{a_{0}\left(n_{0}, t\right)}=b\left(n_{0}+1, t\right)-b_{0}\left(n_{0}+1, t\right)-b\left(n_{0}, t\right)+b_{0}\left(n_{0}, t\right) \\
& =b\left(n_{0}+1, t\right)-b_{0}\left(n_{0}+1, t\right),
\end{aligned}
$$

so $b\left(n_{0}+1, t\right)=b_{0}\left(n_{0}+1, t\right)$. Now, use that

$$
\begin{aligned}
0 & =\dot{b}\left(n_{0}+1, t\right)-\dot{b}_{0}\left(n_{0}+1, t\right) \\
& =2\left(a\left(n_{0}+1, t\right)^{2}-a_{0}\left(n_{0}+1, t\right)^{2}-a\left(n_{0}, t\right)^{2}+a_{0}\left(n_{0}, t\right)^{2}\right) \\
& =2\left(a\left(n_{0}+1, t\right)^{2}-a_{0}\left(n_{0}+1, t\right)^{2}\right)
\end{aligned}
$$

to conclude that $a\left(n_{0}+1, t\right)^{2}=a_{0}\left(n_{0}+1, t\right)^{2}$. This finishes the proof. 
It is worthwhile to note that the assumption $a_{0}(n, t) \neq 0$ is crucial. In fact, if $a_{0}\left(n_{0}, t\right)=0$ for one (and hence for all) $t \in \mathbb{R}$, then the Toda lattice decouples into two independent parts to the left and right of $n_{0}$, and the above result is clearly wrong. However, it remains valid on every consecutive number of points for which $a_{0}(n, t) \neq 0$ holds true. In particular, our result applies to the half-line Toda lattice or to the finite Toda lattice.

As a simple consequence, this also proves that the propagation speed is finite for the Toda lattice.

Corollary 2.2. Let $a(n, t) \neq 0$, and let $b(n, t)$ be a complex-valued solution of the Toda lattice (2.1) for which $a\left(n, t_{0}\right)-\frac{1}{2}, b\left(n, t_{0}\right)$ is supported on a finite number of points $n$ at some initial time $t_{0}$. Then this does not remain true for $t \in\left(t_{0}, t_{1}\right)$ unless $a(n, t)=\frac{1}{2}, b(n, t)=0$ for all $(n, t) \in \mathbb{Z} \times \mathbb{R}$.

In fact, in the case of real-valued solutions, one can even show the somewhat stronger result that $a\left(n, t_{0}\right)-\frac{1}{2}, b\left(n, t_{0}\right)$ can be compactly supported for at most one time [18]. However, on the other hand, the Toda lattice does preserve certain asymptotic properties of the initial conditions; see again [18].

\section{Extension to the Toda and KaC-Van Moerbeke hierarchy}

In this section we show that our main result extends to the entire Toda hierarchy (which will cover the Kac-van Moerbeke hierarchy as well). To this end, we introduce the Toda hierarchy using the standard Lax formalism following [4] (see also [9], [16]).

Associated with two sequences $a(t)^{2} \neq 0, b(t)$ is a Jacobi operator

$$
H(t)=a(t) S^{+}+a^{-}(t) S^{-}+b(t)
$$

acting on sequences over $\mathbb{Z}$, where $S^{ \pm} f(n)=f^{ \pm}(n)=f(n \pm 1)$ are the usual shift operators. Moreover, choose constants $c_{0}=1, c_{j}, 1 \leq j \leq r, c_{r+1}=0$, and set

$$
P_{2 r+2}(t)=\sum_{j=0}^{r} c_{r-j} \tilde{P}_{2 j+2}(t), \quad \tilde{P}_{2 j+2}(t)=\left[H(t)^{j+1}\right]_{+}-\left[H(t)^{j+1}\right]_{-},
$$

where $[A]_{ \pm}$denote the upper and lower triangular parts of an operator with respect to the standard basis $\delta_{m}(n)=\delta_{m, n}$ (with $\delta_{m, n}$ the usual Kronecker delta). Then the Toda hierarchy is equivalent to the Lax equation

$$
\frac{d}{d t} H(t)-\left[P_{2 r+2}(t), H(t)\right]=0, \quad t \in \mathbb{R},
$$

where $[A, B]=A B-B A$ is the usual commutator. Abbreviating

$$
\begin{aligned}
& g_{j}(n, t)=\sum_{\ell=0}^{j} c_{j-\ell} \tilde{g}_{\ell}(n, t), \quad \tilde{g}_{\ell}(n, t)=\left\langle\delta_{n}, H(t)^{\ell} \delta_{n}\right\rangle, \\
& h_{j}(n, t)=2 a(n, t) \sum_{\ell=0}^{j} c_{j-\ell} \tilde{h}_{\ell}(n, t)+c_{j+1}, \quad \tilde{h}_{\ell}(n, t)=\left\langle\delta_{n+1}, H(t)^{\ell} \delta_{n}\right\rangle,
\end{aligned}
$$

one explicitly obtains

$$
\mathrm{TL}_{r}(a(t), b(t))=\left(\begin{array}{c}
\dot{a}(t)-a(t)\left(g_{r+1}^{+}(t)-g_{r+1}(t)\right) \\
\dot{b}(t)-\left(h_{r+1}(t)-h_{r+1}^{-}(t)\right)
\end{array}\right)=0, \quad r \in \mathbb{N}_{0}
$$


for the $r$-th equation $\mathrm{TL}_{r}(a, b)=0$ in the Toda hierarchy (where $\mathbb{N}_{0}=\mathbb{N} \cup\{0\}$ ).

Our main point in this section is the following generalization of Theorem 2.1 to the entire Toda hierarchy:

Theorem 3.1. Assume that $a_{0}(n, t) \neq 0, b_{0}(n, t)$ and $a(n, t), b(n, t)$ are complexvalued solutions of some equation in the Toda hierarchy $\mathrm{TL}_{r}$ such that there is one $n_{0} \in \mathbb{Z}$ and two times $t_{0}<t_{1}$ such that

$$
a_{0}\left(n_{0}+j, t\right)^{2}=a\left(n_{0}+j, t\right)^{2}, \quad b_{0}\left(n_{0}+j, t\right)=b\left(n_{0}+j, t\right), \quad j=0, \ldots, r,
$$

for $t \in\left(t_{0}, t_{1}\right)$. Then

$$
a_{0}(n, t)^{2}=a(n, t)^{2}, \quad b_{0}(n, t)=b(n, t)
$$

for all $(n, t) \in \mathbb{Z} \times \mathbb{R}$.

Proof. Let us drop the dependence on $t$ for notational simplicity during this proof. The key observation is the following structure for the homogenous quantities $\tilde{g}_{j}$, $\tilde{h}_{j}$ :

$$
\tilde{g}_{j}(n)=\left\{\begin{aligned}
\left(\prod_{\ell=0}^{k-1} a(n+\ell)^{2}\right) b(n+k)+R(n+k-1, n-k+1) & \\
+\left(\prod_{\ell=1}^{k} a(n-\ell)^{2}\right)\left(b(n-k)+2 \sum_{\ell=0}^{k-1} b(n-\ell)\right), & j=2 k+1, \\
\left(\prod_{\ell=0}^{k-2} a(n+\ell)^{2}\right)\left(a(n+k-1)^{2}+b(n+k-1)^{2}\right. & \\
\left.+2 b(n+k-1) \sum_{\ell=0}^{k-2} b(n+\ell)\right) & \\
+R(n+k-2, n-k+1)+\prod_{\ell=1}^{k} a(n-\ell)^{2}, & j=2 k,
\end{aligned}\right.
$$

and

$$
\tilde{h}_{j}(n)=\left\{\begin{aligned}
& 2\left(\prod_{\ell=0}^{k-1} a(n+\ell)^{2}\right)\left(a(n+k)^{2}+b(n+k)^{2}\right. \\
&\left.+2 b(n+k) \sum_{\ell=0}^{k-1} b(n+\ell)\right) \\
&+ R(n+k-1, n-k+1)+2 \prod_{\ell=0}^{k} a(n-\ell)^{2}, \\
& 2\left(\prod_{\ell=0}^{k-1} a(n+\ell)^{2}\right) b(n+k)+R(n+k-1, n-k+2) \\
&+2 \prod_{\ell=0}^{k-1} a(n-\ell)^{2}\left(b(n+1)+b(n-k+1)+2 \sum_{\ell=0}^{k-2} b(n-\ell)\right), \quad j=2 k,
\end{aligned}\right.
$$

for $j>1$. Here $R(n, m)$ denotes terms which involve only $a(\ell)$ and $b(\ell)$ with $m \leq \ell \leq n$ and we set $R(n, m)=0$ if $n<m$. In fact, this can be verified using $\tilde{g}_{0}=1, \tilde{\tilde{h}}_{0}=0$, together with the recursions ([16, Chap. 6])

$$
\begin{aligned}
& \tilde{g}_{j+1}=\frac{\tilde{h}_{j}+\tilde{h}_{j}^{-}}{2}+b \tilde{g}_{j}, \\
& \tilde{h}_{j+1}=2 a^{2} \sum_{\ell=0}^{j} \tilde{g}_{j-\ell} \tilde{g}_{\ell}^{+}-\frac{1}{2} \sum_{\ell=0}^{j} \tilde{h}_{j-\ell} \tilde{h}_{\ell}, \quad j \in \mathbb{N}_{0} .
\end{aligned}
$$


Now we are ready for the main part of the proof. It suffices to show that (3.7) holds for $n=n_{0}-1$ and $n=n_{0}+r+1$.

We first look at the case $r+1=2 k+1$. Then

$$
\begin{aligned}
0 & =\frac{\dot{a}\left(n_{0}+k\right)}{a\left(n_{0}+k\right)}-\frac{\dot{a}_{0}\left(n_{0}+k\right)}{a_{0}\left(n_{0}+k\right)} \\
& =g_{r+1}\left(n_{0}+k+1\right)-g_{r+1}\left(n_{0}+k\right)-g_{0, r+1}\left(n_{0}+k+1\right)+g_{0, r+1}\left(n_{0}+k\right) \\
& =\left(\prod_{\ell=k+1}^{r} a_{0}\left(n_{0}+\ell\right)^{2}\right)\left(b\left(n_{0}+r+1\right)-b_{0}\left(n_{0}+r+1\right)\right)
\end{aligned}
$$

shows that $b\left(n_{0}+r+1\right)=b_{0}\left(n_{0}+r+1\right)$. Similarly,

$$
\begin{aligned}
0 & =\dot{b}\left(n_{0}+k\right)-\dot{b}_{0}\left(n_{0}+k\right) \\
& =h_{r+1}\left(n_{0}+k\right)-h_{r+1}\left(n_{0}+k-1\right)-h_{0, r+1}\left(n_{0}+k\right)+h_{0, r+1}\left(n_{0}+k-1\right) \\
& =2\left(\prod_{\ell=0}^{k-1} a_{0}\left(n_{0}+\ell\right)^{2}\right)\left(a\left(n_{0}-1\right)^{2}-a_{0}\left(n_{0}-1\right)^{2}\right)
\end{aligned}
$$

shows that $a\left(n_{0}-1\right)^{2}=a_{0}\left(n_{0}-1\right)^{2}$. Proceeding like this and using the result found in the previous steps,

$$
\begin{aligned}
0 & =\dot{b}\left(n_{0}+k+1\right)-\dot{b}_{0}\left(n_{0}+k+1\right) \\
& =h_{r+1}\left(n_{0}+k+1\right)-h_{r+1}\left(n_{0}+k\right)-h_{0, r+1}\left(n_{0}+k+1\right)+h_{0, r+1}\left(n_{0}+k\right) \\
& =\left(\prod_{\ell=k+1}^{r} a_{0}\left(n_{0}+\ell\right)^{2}\right)\left(a\left(n_{0}+r+1\right)^{2}-a_{0}\left(n_{0}+r+1\right)^{2}\right)
\end{aligned}
$$

shows that $a\left(n_{0}+r+1\right)^{2}=a_{0}\left(n_{0}+r+1\right)^{2}$, and

$$
\begin{aligned}
0 & =\frac{\dot{a}\left(n_{0}+k-1\right)}{a\left(n_{0}+k-1\right)}-\frac{\dot{a}_{0}\left(n_{0}+k-1\right)}{a_{0}\left(n_{0}+k-1\right)} \\
& =g_{r+1}\left(n_{0}+k\right)-g_{r+1}\left(n_{0}+k-1\right)-g_{0, r+1}\left(n_{0}+k\right)+g_{0, r+1}\left(n_{0}+k-1\right) \\
& =\left(\prod_{\ell=-1}^{k} a_{0}\left(n_{0}+\ell\right)^{2}\right)\left(b\left(n_{0}-1\right)-b_{0}\left(n_{0}-1\right)\right)
\end{aligned}
$$

shows that $b\left(n_{0}-1\right)=b_{0}\left(n_{0}-1\right)$, which finishes the case $r+1=2 k+1$. The case $r+1=2 k$ is analogous.

Finally, since the Kac-van Moerbeke hierarchy can be obtained by setting $b=0$ in the odd equations of the Toda hierarchy, $\operatorname{KM}_{r}(a)=\mathrm{TL}_{2 r+1}(a, 0)$ (see [15), this last result also covers the Kac-van Moerbeke hierarchy. In particular,

Corollary 3.2. Assume that $\rho_{0}(n, t) \neq 0$ and $\rho(n, t) \neq 0$ are solutions of the Kac-van Moerbeke equation

$$
\dot{\rho}(n, t)=\rho(n, t)(\rho(n+1, t)-\rho(n-1, t))
$$

such that there is one $n_{0} \in \mathbb{Z}$ and two times $t_{0}<t_{1}$ such that

$$
\rho_{0}\left(n_{0}, t\right)=\rho\left(n_{0}, t\right), \quad \rho_{0}\left(n_{0}+1, t\right)=\rho\left(n_{0}+1, t\right),
$$


for $t \in\left(t_{0}, t_{1}\right)$. Then

$$
\rho_{0}(n, t)=\rho(n, t)
$$

for all $(n, t) \in \mathbb{Z} \times \mathbb{R}$.

\section{The AblowitZ-Ladik hierarchy}

In this section we show that our main result extends to the Ablowitz-Ladik (AL) hierarchy [9]. We first state the result for the simplest case, whose proof follows as the one of Theorem 2.1

Theorem 4.1. Let $C_{0, \pm}, c_{1} \in \mathbb{C} \backslash\{0\}$. Assume that $\alpha_{0}(n, t), \beta_{0}(n, t)$, with $\rho_{0}(n, t) \neq$ 0 , and $\alpha(n, t), \beta(n, t)$ are solutions of the Ablowitz-Ladik equation

$$
\begin{aligned}
& \mathrm{i} \dot{\alpha}(n, t)=-\rho(n, t)^{2}\left(c_{0,-} \alpha(n-1, t)+c_{0,+} \alpha(n+1, t)\right)-c_{1} \alpha(n, t), \\
& \mathrm{i} \dot{\beta}(n, t)=\rho(n, t)^{2}\left(c_{0,+} \beta(n-1, t)+c_{0,-} \beta(n+1, t)\right)+c_{1} \beta(n, t),
\end{aligned}
$$

where

$$
\rho(n, t)=(1-\alpha(n, t) \beta(n, t))^{1 / 2},
$$

such that there is one $n_{0} \in \mathbb{Z}$ and two times $t_{0}<t_{1}$ such that

$$
\alpha_{0}\left(n_{0}+j, t\right)=\alpha\left(n_{0}+j, t\right), \quad \beta_{0}\left(n_{0}+j, t\right)=\beta\left(n_{0}+j, t\right), \quad j=0,1,
$$

for $t \in\left(t_{0}, t_{1}\right)$. Then

$$
\alpha_{0}(n, t)=\alpha(n, t), \quad \beta_{0}(n, t)=\beta(n, t)
$$

for all $(n, t) \in \mathbb{Z} \times \mathbb{R}$.

The special choices $c_{0, \pm}=1, c_{1}=-2$, and $\beta= \pm \bar{\alpha}$ yield the focusing, defocusing discrete nonlinear Schrödinger equations, respectively. The alternative choice $c_{0, \pm}= \pm \mathrm{i}, c_{1}=0$, and $\beta=\bar{\alpha}$ yield the Schur flow.

We next turn to the AL hierarchy following [8], 9]. Associated with two sequences $\alpha(t), \beta(t)$ is a CMV operator

$$
\begin{aligned}
L(t)= & \rho^{-}(t) \rho(t) \delta_{\text {even }} S^{--}+\left(\beta^{-}(t) \rho(t) \delta_{\text {even }}-\alpha^{+}(t) \rho(t) \delta_{\text {odd }}\right) S^{-}-\beta(t) \alpha^{+}(t) \\
& +\left(\beta(t) \rho^{+}(t) \delta_{\text {even }}-\alpha^{++}(t) \rho^{+}(t) \delta_{\text {odd }}\right) S^{+}+\rho^{+}(t) \rho^{++}(t) \delta_{\text {odd }} S^{++},
\end{aligned}
$$

acting on sequences over $\mathbb{Z}$, where $\delta_{\text {even }}$ and $\delta_{\text {odd }}$ denote the characteristic functions of the even, odd integers,

$$
\delta_{\text {even }}=\chi_{2 \mathbb{Z}}, \quad \delta_{\text {odd }}=1-\delta_{\text {even }}=\chi_{2 \mathbb{Z}+1},
$$

respectively. Next, consider

$$
\begin{aligned}
P_{\underline{p}}(t)= & \frac{\mathrm{i}}{2} \sum_{\ell=1}^{p_{+}} c_{p_{+}-\ell,+}\left(\left[L^{\ell}(t)\right]_{+}-\left[L^{\ell}(t)\right]_{-}\right) \\
& -\frac{\mathrm{i}}{2} \sum_{\ell=1}^{p_{-}} c_{p_{-}-\ell,-}\left(\left[L^{-\ell}(t)\right]_{+}-\left[L^{-\ell}(t)\right]_{-}\right)-\frac{\mathrm{i}}{2} c_{\underline{p}} Q_{d}, \quad \underline{p} \in \mathbb{N}_{0}^{2},
\end{aligned}
$$

with $Q_{d}$ denoting the doubly infinite diagonal matrix

$$
Q_{d}=\left((-1)^{k} \delta_{k, \ell}\right)_{k, \ell \in \mathbb{Z}} .
$$

Then the AL hierarchy is equivalent to the Lax equation

$$
\frac{d}{d t} L(t)-\left[P_{\underline{p}}(t), L(t)\right]=0, \quad t \in \mathbb{R} .
$$


To find an explicit expression we introduce

$$
\begin{aligned}
f_{\ell, \pm}(t) & =\sum_{k=0}^{\ell} c_{\ell-k, \pm} \hat{f}_{k, \pm}(t), \quad g_{\ell, \pm}(t)=\sum_{k=0}^{\ell} c_{\ell-k, \pm} \hat{g}_{k, \pm}(t), \\
h_{\ell, \pm}(t) & =\sum_{k=0}^{\ell} c_{\ell-k, \pm} \hat{h}_{k, \pm}(t),
\end{aligned}
$$

where

$$
\begin{aligned}
& \hat{f}_{\ell,+}(n, t)=\alpha(n, t)\left\langle\delta_{n}, L^{\ell+1} \delta_{n}\right\rangle+\rho(n, t) \begin{cases}\left\langle\delta_{n-1}, L^{\ell+1}(t) \delta_{n}\right\rangle, & n \text { even }, \\
\left\langle\delta_{n}, L^{\ell+1}(t) \delta_{n-1}\right\rangle, & n \text { odd },\end{cases} \\
& \hat{f}_{\ell,-}(n, t)=\alpha(n, t)\left(\delta_{n}, L^{-\ell} \delta_{n}\right)+\rho(n, t) \begin{cases}\left\langle\delta_{n-1}, L^{-\ell}(t) \delta_{n}\right\rangle, & n \text { even, } \\
\left\langle\delta_{n}, L^{-\ell}(t) \delta_{n-1}\right\rangle, & n \text { odd, }\end{cases} \\
& \hat{g}_{0, \pm}=1 / 2, \quad \hat{g}_{\ell, \pm}(n, t)=\left\langle\delta_{n}, L^{ \pm \ell}(t) \delta_{n}\right\rangle \text {, } \\
& \hat{h}_{\ell,+}(n, t)=\beta(n, t)\left\langle\delta_{n}, L^{\ell}(t) \delta_{n}\right\rangle+\rho(n, t) \begin{cases}\left\langle\delta_{n}, L^{\ell}(t) \delta_{n-1}\right\rangle, & n \text { even, } \\
\left\langle\delta_{n-1}, L^{\ell}(t) \delta_{n}\right\rangle, & n \text { odd, }\end{cases} \\
& \hat{h}_{\ell,-}(n, t)=\beta(n, t)\left\langle\delta_{n}, L^{-\ell-1} \delta_{n}\right\rangle+\rho(n, t) \begin{cases}\left\langle\delta_{n}, L^{-\ell-1}(t) \delta_{n-1}\right\rangle, & n \text { even, } \\
\left\langle\delta_{n-1}, L^{-\ell-1}(t) \delta_{n}\right\rangle, & n \text { odd }\end{cases}
\end{aligned}
$$

Then the $\underline{p}$ th equation, $\underline{p}=\left(p_{-}, p_{+}\right) \in \mathbb{N}_{0}^{2}$, in the AL hierarchy is given by

$$
\begin{gathered}
\mathrm{AL}_{\underline{p}}(\alpha, \beta)=\left(\begin{array}{c}
-\mathrm{i} \dot{\alpha}(t)-\alpha\left(g_{p_{+},+}(t)+g_{p_{-},-}^{-}(t)\right)+f_{p_{+}-1,+}(t)-f_{p_{-}-1,-}^{-}(t) \\
-\mathrm{i} \dot{\beta}(t)+\beta\left(g_{p_{+},+}^{-}(t)+g_{p_{-},}(t)\right)-h_{p_{-}-1,-}(t)+h_{p_{+}-1,+}^{-}(t)
\end{array}\right)=0, \\
\underline{p}=\left(p_{-}, p_{+}\right) \in \mathbb{N}_{0}^{2} .
\end{gathered}
$$

Theorem 4.2. Fix some $\underline{p}=\left(p_{+}, p_{-}\right) \in \mathbb{N}_{0}^{2}$ such that $p_{-}=p_{+}>0$ and set $p=p_{+}+p_{-}-1$. Assume that $\alpha_{0}(n, t), \beta_{0}(n, t)$, with $\rho_{0}(n, t) \neq 0$, and $\alpha(n, t), \beta(n, t)$ are solutions of some equation in the Toda hierarchy $\mathrm{AL}_{p}$ such that there is one $n_{0} \in \mathbb{Z}$ and two times $t_{0}<t_{1}$ such that

$$
\alpha_{0}\left(n_{0}+j, t\right)=\alpha\left(n_{0}+j, t\right), \quad \beta_{0}\left(n_{0}+j, t\right)=\beta\left(n_{0}+j, t\right), \quad 0 \leq j \leq p,
$$

for $t \in\left(t_{0}, t_{1}\right)$. Then

$$
\alpha_{0}(n, t)=\alpha(n, t), \quad \beta_{0}(n, t)=\beta(n, t)
$$

for all $(n, t) \in \mathbb{Z} \times \mathbb{R}$.

Proof. Again we drop the dependence on $t$ for notational simplicity during this proof and use the same conventions as in the proof of Theorem 3.1 
The homogeneous quantities $\hat{f}_{\ell, \pm}, \hat{g}_{\ell, \pm}, \hat{h}_{\ell, \pm}$ are uniquely defined by the following recursion relations [9, Lem. C.5]:

$$
\begin{aligned}
\hat{g}_{0,+} & =\frac{1}{2}, \quad \hat{f}_{0,+}=-\alpha^{+}, \quad \hat{h}_{0,+}=\beta, \\
\hat{g}_{l+1,+} & =\sum_{k=0}^{l} \hat{f}_{l-k,+} \hat{h}_{k,+}-\sum_{k=1}^{l} \hat{g}_{l+1-k,+} \hat{g}_{k,+}, \\
\hat{f}_{l+1,+}^{-} & =\hat{f}_{l,+}-\alpha\left(\hat{g}_{l+1,+}+\hat{g}_{l+1,+}^{-}\right), \\
\hat{h}_{l+1,+} & =\hat{h}_{l,+}^{-}+\beta\left(\hat{g}_{l+1,+}+\hat{g}_{l+1,+}^{-}\right),
\end{aligned}
$$

and

$$
\begin{aligned}
\hat{g}_{0,-} & =\frac{1}{2}, \quad \hat{f}_{0,-}=\alpha, \quad \hat{h}_{0,-}=-\beta^{+}, \\
\hat{g}_{l+1,-} & =\sum_{k=0}^{l} \hat{f}_{l-k,-} \hat{h}_{k,-}-\sum_{k=1}^{l} \hat{g}_{l+1-k,-} \hat{g}_{k,-}, \\
\hat{f}_{l+1,-} & =\hat{f}_{l,-}^{-}+\alpha\left(\hat{g}_{l+1,-}+\hat{g}_{l+1,-}^{-}\right), \\
\hat{h}_{l+1,-}^{-} & =\hat{h}_{l,-}-\beta\left(\hat{g}_{l+1,-}+\hat{g}_{l+1,-}^{-}\right) .
\end{aligned}
$$

From them we obtain

$$
\begin{aligned}
\hat{f}_{j,+}(n)= & -\left(\prod_{l=1}^{j} \rho(n+l)^{2}\right) \alpha(n+j+1)+R(n+j, n-j+2) \\
& +\left(\prod_{l=0}^{j-2} \rho(n-l)^{2}\right) \alpha(n+1)^{2} \beta(n-j+1), \\
\hat{f}_{j,-}(n)= & -\left(\prod_{l=1}^{j-1} \rho(n+l)^{2}\right) \alpha(n)^{2} \beta(n+j)+R(n+j-1, n-j+1) \\
& +\left(\prod_{l=0}^{j-1} \rho(n-l)^{2}\right) \alpha(n-j), \\
\hat{g}_{j,+}(n)= & -\left(\prod_{l=1}^{j-1} \rho(n+l)^{2}\right) \beta(n) \alpha(n+j)+R(n+j-1, n-j+2) \\
& -\left(\prod_{l=0}^{j-2} \rho(n-l)^{2}\right) \alpha(n+1) \beta(n-j+1), \\
\hat{g}_{j,-}(n)= & -\left(\prod_{l=1}^{j-1} \rho(n+l)^{2}\right) \alpha(n) \beta(n+j)+R(n+j-1, n-j+2) \\
& -\left(\prod_{l=0}^{j-2} \rho(n-l)^{2}\right) \beta(n+1) \alpha(n-j+1),
\end{aligned}
$$




$$
\begin{aligned}
\hat{h}_{j,+}(n)= & -\left(\prod_{l=1}^{j-1} \rho(n+l)^{2}\right) \beta(n)^{2} \alpha(n+j)+R(n+j-1, n-j+1) \\
& +\left(\prod_{l=0}^{j-1} \rho(n-l)^{2}\right) \beta(n-j), \\
\hat{h}_{j,-}(n)= & -\left(\prod_{l=1}^{j} \rho(n+l)^{2}\right) \beta(n+j+1)+R(n+j, n-j+2) \\
& +\left(\prod_{l=0}^{j-2} \rho(n-l)^{2}\right) \beta(n+1)^{2} \alpha(n-j+1)
\end{aligned}
$$

for $j \in \mathbb{N}$. Note that it suffices to verify the + case since the - case follows from $\hat{f}_{j, \pm}(\alpha, \beta)=\hat{h}_{j, \pm}(\alpha, \beta)$ and $\hat{g}_{j,+}(\alpha, \beta)=\hat{g}_{j,}(\alpha, \beta)([9$, Lem. 3.7]).

Now we can proceed as in the case of the Toda hierarchy. For example,

$0=\mathrm{i}\left(\dot{\alpha}\left(n+p_{-}\right)-\dot{\alpha}_{0}\left(n+p_{-}\right)\right)=-c_{0,+}\left(\prod_{l=p_{-}}^{p} \rho(n-l)^{2}\right)\left(\alpha(n+p+1)-\alpha_{0}(n+p+1)\right)$

implies that $\alpha(n+p+1)=\alpha_{0}(n+p+1)$, etc.

Interestingly, the above approach does not seem to work for $p_{-} \neq p_{+}$in general. In any case, the above result covers the discrete nonlinear Schrödinger and Schur hierarchies via the above-mentioned special choices $c_{0, \pm}=1, \beta= \pm \bar{\alpha}$ and $c_{0, \pm}= \pm \mathrm{i}$, $\beta=\bar{\alpha}$.

\section{ACKNOWLEDGMEnts}

We thank F. Gesztesy and the anonymous referee for pointing out errors in a previous version of this article.

\section{REFERENCES}

[1] J. Bourgain, On the compactness of the support of solutions of dispersive equations, Internat. Math. Res. Notices 1997, no. 9, 437-447 (1997). MR.1443322 (98a:35113)

[2] J. Bourgain, Anderson-Bernoulli models, Mosc. Math. J., 5:3, 523-536 (2005). MR2241811 (2007f:82042)

[3] J. Bourgain and C. E. Kenig, On localization in the continuous Anderson-Bernoulli model in higher dimension, Invent. Math. 161:2, 389-426 (2005). MR2180453 (2006k:82085)

[4] W. Bulla, F. Gesztesy, H. Holden, and G. Teschl, Algebro-Geometric Quasi-Periodic FiniteGap Solutions of the Toda and Kac-van Moerbeke Hierarchies, Mem. Amer. Math. Soc. 135:641 (1998). MR1432141 (99b:58109)

[5] L. Escauriaza, C. E. Kenig, G. Ponce, and L. Vega, On uniqueness properties of solutions of Schrödinger equations, Comm. Partial Differential Equations 31:12, 1811-1823 (2006). MR2273975 (2009a:35198)

[6] L. Faddeev and L. Takhtajan, Hamiltonian Methods in the Theory of Solitons, Springer, Berlin, 1987. MR905674 (89m:58103)

[7] H. Flaschka, The Toda lattice. I. Existence of integrals, Phys. Rev. B 9, 1924-1925 (1974). MR0408647 (53:12411)

[8] F. Gesztesy, H. Holden, J. Michor, and G. Teschl, The Ablowitz-Ladik hierarchy revisited, in "Methods of Spectral Analysis in Mathematical Physics", J. Janas et al. (eds.), 139-190, Oper. Theory Adv. Appl. 186, Birkhäuser, Basel, 2009. MR2732077 
[9] F. Gesztesy, H. Holden, J. Michor, and G. Teschl, Soliton Equations and Their AlgebroGeometric Solutions. Volume II: $(1+1)$-Dimensional Discrete Models, Cambridge Studies in Advanced Mathematics 114, Cambridge University Press, Cambridge, 2008. MR2446594 (2011b:37130)

[10] A. Ionescu and C. E. Kenig, $L^{p}$ Carleman inequalities and uniqueness of solutions of nonlinear Schrödinger equations, Acta Math. 193:2, 193-239 (2004). MR2134866 (2005m:35283)

[11] C. E. Kenig, G. Ponce, and L. Vega, On the support of solutions to the generalized KdV equation, Ann. Inst. Henri Poincaré 19:2, 191-208 (2002). MR.1902743 (2003j:35275)

[12] C. E. Kenig, G. Ponce, and L. Vega, On unique continuation of solutions to the generalized KdV equation, Math. Res. Lett. 10, 833-846 (2003). MR2025059 (2005e:35201)

[13] H. Krüger and G. Teschl, Long-time asymptotics for the Toda lattice for decaying initial data revisited, Rev. Math. Phys. 21:1, 61-109 (2009). MR2493113 (2011b:37144)

[14] J. Lenells, Infinite propagation speed of the Camassa-Holm equation, J. Math. Anal. Appl. 325:2, 1468-1478 (2007). MR2275032 (2007h:35286)

[15] J. Michor and G. Teschl, On the equivalence of different Lax pairs for the Kac-van Moerbeke hierarchy, in Modern Analysis and Applications, V. Adamyan et al. (eds.), 445-453, Oper. Theory Adv. Appl. 191, Birkhäuser, Basel, 2009. MR2569413 (2011b:37148)

[16] G. Teschl, Jacobi Operators and Completely Integrable Nonlinear Lattices, Math. Surv. and Monographs 72, Amer. Math. Soc., Providence, RI, 2000. MR.1711536 (2001b:39019)

[17] G. Teschl, Almost everything you always wanted to know about the Toda equation, Jahresber. Deutsch. Math.-Verein. 103, no. 4, 149-162 (2001). MR1879178 (2002k:37154)

[18] G. Teschl, On the spatial asymptotics of solutions of the Toda lattice, Discrete Contin. Dyn. Syst. 27:3, 1233-1239 (2010). MR2629584

[19] M. Toda, Theory of Nonlinear Lattices, 2nd enlarged ed., Springer, Berlin, 1989. MR971987 (89h:58082)

[20] B. Zhang, Unique continuation for the Korteweg-de Vries equation, SIAM J. Math. Anal. 23, 55-71 (1992). MR:1145162 (92k:35252)

Department of Mathematics, Rice University, Houston, Texas 77005

Current address: Department of Mathematics, California Institute of Technology, Pasadena, California 91125

E-mail address: helge@caltech.edu

URL: http://www.its.caltech.edu/ ^helge/

Faculty of Mathematics, University of Vienna, Nordbergstrasse 15, 1090 Wien, Austria - And - International ERWin Schrödinger Institute for Mathematical Physics, Boltzmanngasse 9, 1090 Wien, Austria

E-mail address: Gerald.Teschl@univie.ac.at

URL: http://www.mat.univie.ac.at/〜gerald/ 